\title{
IDENTITIES IN FINITE PARTITION LATTICES
}

\section{DAVID SACHS}

In his discussion of the problem of imbedding a finite lattice into a finite partition lattice, Birkhoff [1] speculates that there are no nontrivial identities satisfied in every finite partition lattice. We give a simple proof of this conjecture based upon Whitman's Theorem [4]. Since a partition lattice $P$ is a complete, meet-continuous lattice in which every element is a join of points [3], $P$ is isomorphic to the lattice of ideals of its sublattice $P^{\prime}$ of finite-dimensional elements. We prove Birkhoff's conjecture by focusing attention on $P^{\prime}$ rather than on $P$ directly.

We denote the join and meet operations by + and $\cdot$. A lattice polynomial form will be denoted by $f\left(x_{1}, \cdots, x_{1}, \cdots, x_{n}, \cdots, x_{n}\right)$, where we repeat a variable $m$ times if it appears $m$ times in the form.

Lemma. If an identity is valid in a lattice $L$, then it is valid in the lattice $L^{\prime}$ of all ideals of $L$.

Proof. Let the identity be

$$
\begin{aligned}
& f\left(x_{1}, \cdots, x_{1}, \cdots, x_{n}, \cdots, x_{n}\right) \\
& =g\left(x_{1}, \cdots, x_{1}, \cdots, x_{n}, \cdots, x_{n}\right) .
\end{aligned}
$$

If $t \in f\left(X_{1}, \cdots, X_{1}, \cdots, X_{n}, \cdots, X_{n}\right)$, then

$$
t \leqq f\left(x_{11}, \cdots, x_{1 i}, \cdots, x_{n 1}, \cdots, x_{n j}\right)
$$

where $x_{p q} \in X_{p}$, the $X_{p}$ being ideals. Obviously

$$
\begin{aligned}
t & \leqq f\left(x_{1}^{\prime}, \cdots, x_{1}^{\prime}, \cdots, x_{n}{ }^{\prime}, \cdots, x_{n}{ }^{\prime}\right) \\
& =g\left(x_{1}^{\prime}, \cdots, x_{1}^{\prime}, \cdots, x_{n}^{\prime}, \cdots, x_{n}{ }^{\prime}\right)
\end{aligned}
$$

where $x_{t}^{\prime}=\sum_{p} x_{t p}$.

Thus $t \in g\left(X_{1}, \cdots, X_{1}, \cdots, X_{n}, \cdots, X_{n}\right)$ since the $X_{p}$ are ideals. It therefore follows that $f\left(X_{1}, \cdots, X_{1}, \cdots, X_{n}, \cdots, X_{n}\right)$ $\subseteq g\left(X_{1}, \cdots, X_{1}, \cdots, X_{n}, \cdots, X_{n}\right)$, and by symmetry the identity $f=g$ is valid in $L^{\prime}$.

REMARK. This lemma is an exercise (unanswered) in [2, p. 80].

THEOREM. Any identity valid in every finite partition lattice is trivial, i.e. is valid in every lattice.

Proof. Let us suppose that the identity $f=g$ is valid in every finite

Received by the editors November 7, 1960. 
partition lattice. Let $P$ be any partition lattice, and let $P^{\prime}$ be its sublattice of finite-dimensional elements. The join of any $n$ elements in $P^{\prime}$ lies in an interval sublattice $[0, a]$ which is isomorphic to a finite partition lattice. (Take the union of all the sets of the $n$ partitions which are nonsingletons.) Thus if the identity $f=g$ is valid in every finite partition lattice, it is valid in $P^{\prime}$. In view of the lemma and the fact that $P$ is isomorphic to the lattice of ideals in $P^{\prime}$, the identity $f=g$ is valid in $P$. By Whitman's Theorem every lattice is a sublattice of some partition lattice, and therefore the identity $f=g$ is valid in every lattice.

COROllary. There are no nontrivial identities valid in any infinite partition lattice.

Corollary. For every nontrivial identity $f=g$, there exists a finite lattice in which it is invalid.

The last corollary suggests the following question: If an identity is valid in every finite sublattice of a lattice $L$, is it valid in $L$ ? This is certainly the case for the modular and distributive identities.

\section{BIBLIOGRAPHY}

1. G. Birkhoff, Some problems of lattice theory, International Congress of Mathematicians, 1950, vol. II, pp. 4-7. 1948.

2. - Lattice theory, Amer. Math. Soc. Colloquium Publications, vol. 25,

3. M. L. Dubreil-Jacotin, L. Lesieur and R. Croisot, Theorie des treillis, Paris, Gauthier-Villars, 1953.

4. P. M. Whitman, Lattices, equivalence relations, and subgroups, Bull. Amer. Math. Soc. vol. 52 (1946) pp. 507-522.

UNIVERSITY OF ILLINOIS 